\title{
Number of Coronal Bright Points in Different Phases of the Solar Activity Cycle
}

\author{
I. Sattarov ${ }^{1}$, A.A. Pevtsov ${ }^{2}$, N.V. Karachek ${ }^{1}$, and Ch.T. Sherdanov ${ }^{1,3}$ \\ ${ }^{1}$ Tashkent State Pedagogical University, 103 Usif Xos-Hojib St., Tashkent 700100, Uzbekistan, \\ email: isattar@astrin.uzsci.net \\ ${ }^{2}$ National Solar Observatory $\dagger$, Sunspot, NM 88349, USA email: apevtsov@nso.edu \\ ${ }^{3}$ Astronomical Institute AS of Uzbekistan, 33 Astronomicheskaya ul., Tashkent 700052, \\ Uzbekistan email: chori@astrin,uzsci.net
}

Abstract. We present the preliminary results of study of the coronal bright points (BPs) using the SoHO/EIT data. We show that the number of BPs in quiet Sun areas does not vary between 1996 and 1997. In 1998 the BPs number (full disk) increases by about $20 \%$.

\section{Analysis and Results}

We have developed software for automatic identification of the coronal bright points (BPs), and have applied this software to the SoHO/EIT data. BP identification is done using the intensity threshold $\left(\mathrm{I}_{c u t}=I_{A v g}+3 \sigma\right)$ and the area of a potential BP. We remove large scale intensity variations from EIT images using median filter, and compute the average intensity $I_{A v g}$ and its standard deviation $\sigma$. When computing the final $\sigma$, we exclude all pixels which intensity exceeds $\mathrm{I}_{\text {cut }}$. Figure 1 shows the distribution of BPs' brightness in three days near the minimum of sunspot activity computed using $\mathrm{I}_{c u t}=$ $50 \mathrm{DN} /$ pixel. The distributions are very similar to each other and are representative of the quiet Sun at the minimum of sunspot activity. A small peak in the left "wing" of the histograms is caused by the intensity variations in the diffuse base corona. This peak disappears at $\mathrm{I}_{\text {cut }}=80 \mathrm{DN} /$ pixel, and then, the histograms show even more similarity between them.

Table 1 shows the average BPs' numbers for three periods of low sunspot activity on 28 March - 30 April 1996 (total number of EIT images is 446), 18-27 March 1997 (90), and 1-10 February 1998 (140). We have calculated the total number of BPs for the full disk and for a central area (heliocentric distance $\rho<0.5$ ). The daily number of BPs identified in EIT/171, 195, 284, and $304 \AA$ images does not vary significantly during the 35 days in 1996 (not shown). On the other hand, the daily number of BPs in 1997 and 1998 show much larger variations. The variations in BPs' number in 1997 and 1998 are associated with increased magnetic activity at mid-latitudes.

We conclude that:

(a) The number of BPs does not change significantly between 1996 and 1997. From 1997 to 1998 the number of BPs increases by $20 \%$ in the full disk and by $5-10 \%$ in central part $(\rho<0.5)$ of solar disk. The increase is related to the active regions' latitudes: coronal systems associated with the ephemeral active regions, magnetic remnants of decaying regions, and filaments and filament channels.

$\dagger$ Operated by the Association of Universities for Research in Astronomy (AURA, Inc) under cooperative agreement with the National Science Foundation (NSF). 


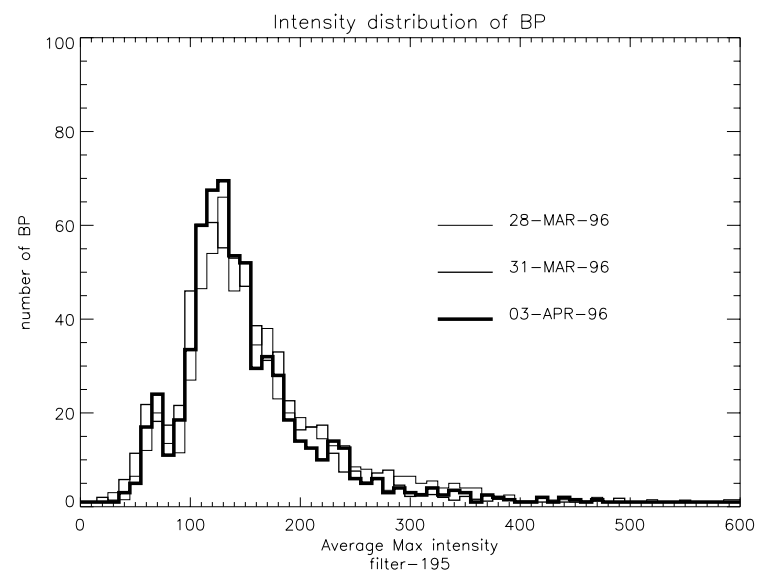

Figure 1. Distribution of the maximum brightness of BPs identified in $195 \AA$ in three different days around the minimum of solar activity.

Table 1. Average number of BPs in four wavelength bands for three different periods.

\begin{tabular}{|c|c|c|}
\hline $\begin{array}{l}\text { EIT band } \AA \\
\text { (No. of images) }\end{array}$ & $\begin{array}{l}\text { Central part } \\
\text { Avg. } \\
\text { No. of BPs }\end{array}$ & $\begin{array}{l}\text { Full disk } \\
\text { Avg. } \\
\text { No. of BPs }\end{array}$ \\
\hline & 1996 & \\
\hline $171(88)$ & $105.7 \pm 9.7$ & $467.7 \pm 31.6$ \\
\hline $195(203)$ & $103.2 \pm 10.7$ & $465.5 \pm 25.0$ \\
\hline $284(72)$ & $121.5 \pm 12.0$ & $533.2 \pm 31.9$ \\
\hline $304(83)$ & $\begin{array}{l}205.6 \pm 11.8 \\
\quad 1997\end{array}$ & $788.1 \pm 24.6$ \\
\hline $171(22)$ & $107.0 \pm 10.5$ & $488.9 \pm 42.7$ \\
\hline $195(22)$ & $101.6 \pm 9.6$ & $457.6 \pm 23.7$ \\
\hline $284(22)$ & $124.5 \pm 10.8$ & $557.2 \pm 55.4$ \\
\hline $304(24)$ & $\begin{array}{c}196.5 \pm 9.3 \\
1998\end{array}$ & $814.0 \pm 23.2$ \\
\hline $171(35)$ & $126.5 \pm 11.4$ & $593.3 \pm 25.7$ \\
\hline $195(35)$ & $117.3 \pm 10.5$ & $599.6 \pm 34.9$ \\
\hline $284(35)$ & $137.1 \pm 11.2$ & $602.5 \pm 78.1$ \\
\hline $304(35)$ & $203.3 \pm 10.9$ & $824.4 \pm 30.1$ \\
\hline
\end{tabular}

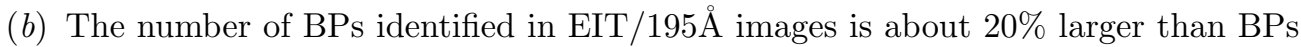
number found by Zhang et al. (2001). Average density of the BPs in our data set is one BP per $81 \mathrm{Mm}^{2}$ (90 $\mathrm{Mm}^{2}$ in Zhang et al., 2001).

\section{Acknowledgements}

Dr. I. Sattarov thanks the IAU's Exchange Astronomers Group and the USA's National Solar Observatory (NSO) for providing travel support for his visit to the NSO at Sacramento Peak.

\section{References}

Zhang, J. Kundu, M.R. \& White, S.M. 2001, Solar Phys. 198, 347. 